\title{
Criminologie
}

\section{La sécurité privée au Canada : quelques questions et réponses}

\section{Clifford D. Shearing}

Volume 17, numéro 1, 1984

La police après 1984

URI : https://id.erudit.org/iderudit/017191ar

DOI : https://doi.org/10.7202/017191ar

Aller au sommaire du numéro

Éditeur(s)

Les Presses de l'Université de Montréal

ISSN

0316-0041 (imprimé)

1492-1367 (numérique)

Découvrir la revue

Citer cet article

Shearing, C. D. (1984). La sécurité privée au Canada : quelques questions et réponses. Criminologie, 17(1), 59-89. https://doi.org/10.7202/017191ar d'utilisation que vous pouvez consulter en ligne.

https://apropos.erudit.org/fr/usagers/politique-dutilisation/ 


\section{LA SÉCURITÉ PRIVÉE AU CANADA: QUELQUES QUESTIONS ET RÉPONSES* \\ C.D. Shearing**}

Ce texte sera consacré à examiner un aspect important et jusqu'ici négligé du processus de maintien de l'ordre: la sécurité privée. La sécurité privée s'est énormément développée au cours des vingt dernières années. Ce phénomène nous atteint tous, d'une façon tranquille mais pénétrante. Dans les édifices à bureaux, les magasins, les universités, les bibliothèques, les centres commerciaux, les maisons de rapport et les subdivisions' ${ }^{\prime}$, nous apercevons souvent et régulièrement des personnes au service de la sécurité privée, qui nous observent à leur tour. Nos contacts avec la sécurité privée se sont multipliés de manière si discrète que cette situation constitue désormais un aspect couramment accepté de la vie moderne. Nous avons tendance à traiter, d'un air quelque peu condescendant, la sécurité privée comme un phénomène extérieur à nos vies, mais il n'en demeure pas moins qu'elle joue un rôle déterminant et constitue un élément essentiel du processus de contrôle social.

De façon chaque fois plus fréquente, la sécurité privée s'immisce dans nos activités; par exemple, lorsqu'on nous arrête à notre sortie d'une bibliothèque pour fouiller nos sacs ou lorsque nous faisons l'objet d'une fouille avant de pénétrer dans la zone d'embarquement dans les aéroports ou encore lorsque nous sommes soumis à des vérifications à l'entrée d'un complexe d'habitation. Occasionnellement, certains gestes posés par une personne au service de la sécurité privée peuvent nous alarmer - par exemple, lorsque nous apprenons que des gardiens de sécurité ont ouvert le feu sur des grévistes militants. Le plus souvent, cependant, la sécurité privée demeure à l'arrière-plan.

* Originellement publié en anglais sous le titre de "Private Security in Canada", in W.T. McGrath et M.P. Mitchell, edit., The Police Function in Canada, Toronto, Methuen Publications, 1981 (copyright 1981 par Methuen Publications). Traduit avec la permission de la maison d'édition.

** Le Docteur Shearing est professeur agrégé et directeur des Études supérieures au Centre de criminologie de l'Université de Toronto. Auparavant, il était membre du Groupe de travail de l'Ontario sur la formation des policiers, instauré par le Solliciteur général de l'Ontario en 1974. Ce texte a été traduit de l'anglais par Sylvie Gagné et Jean-Paul Brodeur.

1. Une «subdivision» est un ensemble de maisons séparées regroupées à l'intérieur d'un mur. Ce mode d'habitation est plus frequent sur la côte ouest du Canada et des États-Unis. Il s'agit en quelque sorte d'une zone d'habitation protégée (n.d.t.). 
Non seulement nos rapports avec la sécurité privée sont-ils à peine perçus mais souvent, c'est même à notre insu qu'ils s'établissent. Ces contacts «invisibles» ont parfois une influence encore plus grande sur nos vies. Ainsi, lorsque nous demandons une ouverture de crédit ou que nous souscrivons à une assurance, nous accordons, de façon typique, à la compagnie concernée la permission de procéder à une "vérification de nos antécédents». Cette enquête est souvent effectuée sans que nous obtenions aucune preuve concrète de ce qui s'est fait ou des conclusions auxquelles on est arrivé.

Il y a toujours eu l'équivalent de ce que nous appelons aujourd'hui la sécurité privée. Ce qui est devenu récemment significatif est l'énorme croissance du secteur de la sécurité privée. Pleece (1972) a ainsi évalué à 20\% par année, le taux d'accroissement des services de sécurité à contrat en Angleterre. De même, Kaklik et Wildhorn (1972) ont constaté pour la sécurité privée aux États-Unis en général, un taux d'accroissement de 10 à 15 pour cent par année. Ils soutiennent que les forces de sécurité privée sont ou seront bientôt plus importantes que les forces de police publique.

Cette progression de la sécurité privée est la conséquence d'une réorganisation fondamentale du contrôle social. Parmi ces mesures de réorganisation le changement le plus visible et, en termes numériques, le plus important est survenu par rapport à la fonction de patrouille. En Angleterre, la «nouvelle police» créée en 1829 fut établie comme un service de patrouille. Depuis lors, la police publique s'est assimilé d'autres composantes et en particulier une fonction d'enquête. Elle est, toutefois, essentiellement demeurée un service de patrouille, bien qu'elle se soit transformée, au cours des cinquante dernières années, d'un service de patrouille à pied en une force motorisée, qui compte sur les appels téléphoniques des citoyens pour être informée des problèmes de ceux-ci (Reiss, 1971; Shearing, 1974).

Cette situation a conduit un sergent de la Police de Northumbria en Angleterre à faire le commentaire suivant: «nous négligeons l'une des pièces d'équipement les plus ingénieuses et les plus efficaces dont la police a jamais disposé: le patrouilleur de quartier, à pied" (cité dans Lewis, 1976, p. 208). Alors que la police négligeait cette "pièce d'équipement", d'autres s'en sont occupés. Le patrouilleur à pied demeure un élément essentiel de notre système de maintien de l'ordre; son poste n'est cependant plus occupé par un policier mais plutôt par un gardien de sécurité privée.

Comme son prédécesseur le patrouilleur de police à pied, le gardien de sécurité privée patrouille pour protéger la propriété et les 
personnes, ainsi que pour assurer le maintien de l'ordre public. En plus de ces similitudes, il existe toutefois d'importantes différences. Ainsi, le gardien de sécurité n'est habituellement pas un agent de la paix et par conséquent, il n'a pas les droits, les pouvoirs et les devoirs d'un policier. De même, contrairement aux policiers qui patrouillent généralement la propriété publique, le gardien de sécurité patrouille habituellement la propriété privée bien qu'il est possible que cette dernière propriété soit d'un accès facile et régulier pour le public, (comme c'est le cas, par exemple, d'un centre d'achats commercial).

L'existence de la sécurité privée soulève des questions quant à ses conséquences pour la qualité de notre vie. Ainsi, quelle signification devons-nous accorder au fait que nous sommes maintenant policés et, en conséquence, surveillés de plus en plus par des forces de sécurité privée employées par des individus ou des institutions privés plutôt que par des gouvernements?

Avant d'aborder de telles questions, il est essentiel d'approfondir notre compréhension du phénomène de la sécurité privée. Au cours de ces dernières années, le Centre de criminologie de l'Université de Toronto a tenté d'élaborer une structure à la fois factuelle et conceptuelle, afin de définir et de comprendre ce qu'est la sécurité privée. Dans le reste de ce texte, nous comptons exploiter ces recherches antérieures dans le but de répondre à certaines questions sur la sécurité privée au Canada.

\section{QU'EST-CE QUE LA SÉCURITÉ PRIVÉE?}

Commençons par une question de définition. En agissant ainsi, nous devons tenter d'élucider les principes qui sous-tendent l'usage courant - ceci n'est pas une tâche facile.

La définition de la sécurité privée a été prise en considération par plusieurs auteurs (voir, par exemple, Kakalik et Wildhorn, 1972 Becker, 1974; Freedman et Stenning, 1977). Aucun, cependant, n'a réussi à formuler une définition également susceptible de distinguer la sécurité privée des autres forces de sécurité et des simples citoyens (private citizens). Un point de départ utile réside dans la définition du concept de sécurité que proposent Freedman et Stenning. Ả l'appui de leur définition, ils citent un haut fonctionnaire de la police en Australie qui a écrit:

La sécurité signifie bien plus que le verrouillage des portes et fenêtres, le transport de l'argent et le déménagement d'objets de valeur. Aujourd'hui, la sécurité est une grosse entreprise commerciale; je fais alors référence non seulement aux services 
de patrouille qui font couramment partie de la scène des grandes villes mais aussi à des services et à des dispositifs tels que des déchiqueteurs de documents, des coffres-forts, des systèmes d'alarme, le transport par véhicules blindés, de l'équipement de communication, des vitres à l'épreuve des balles, des services de courrier, des systèmes d'identification, des enquêtes privées sur l'espionnage industriel et des gardiens armés (1977, p. 9).

En commentant cette déclaration, ils ajoutent les fonctions de sécurité suivantes:

... les agences de renseignement sur le crédit, la surveillance électronique, les polygraphes et autres formes de «détecteur de mensonge», les alarmes contre les incendies, l'équipement pour l'ouverture de lettres soupçonnées d'être piégées, les chiens de garde et même les services exclusivement financiers tels que les cartes de crédit et les chèques de voyage avec leurs slogans publicitaires du genre "Vous ne savez jamais quand vour pourriez être victime d'un vol» $(1977$, p. 9).

La difficulté que présente cette "définition» est qu'elle consiste dans la présentation d'une liste des fonctions de la sécurité plutôt qu'en une définition, nous devons distinguer ce que ces activités ont en commun. Ce qui paraît unifier ces activités est qu'elles se rapportent toutes à la protection de l'information, des biens ou des personnes. Cette conclusion est en accord avec les définitions de la sécurité données par un dictionnaire courant. The Little Oxford Dictionary (1969), par exemple, définit l'adjectif "sûr» (secure) cornme suit: «à l'abri du danger ou de la peur; imprenable, en sûreté...) En un mot, une chose est sûre lorsqu'elle est protégée du danger.

Ayant posé une définition de la sécurité, notre tâche suivante est d'établir la différence entre les diverses catégories de personnes assumant des fonctions de sécurité. À ce sujet, la première distinction à faire est sans doute entre les personnes dont le travail se centre à l'ntérieur de fonctions de sécurité et les personnes qui assument des fonctions de sécurité en dehors du contexte de leurs occupations professionnelles ou dans le prolongement d'un foyer d'occupation qui est autre. Ceci nous permet de distinguer les personnes au service de la sécurité privée des simples citoyens qui remplissent dans le cadre de leurs routines des fonctions de sécurité (fermer les portes à clef, par exemple) et d'autres activités qui se rapportent à de telles fonctions sans être en elles-mêmes des activités de sécurité (ainsi en est-il d'un gérant de banque qui vérifie la porte de la chambre forte avant de quitter son travail). 
Toutefois, même la catégorie «occupation de sécurité» comprend plus que la sécurité privée. Il existe au moins deux importantes occupations de sécurité qui doivent être exclues de notre champ de recherche si nous voulons bien identifier le domaine de la sécurité privée; il s'agit de la police militaire et de la police publique. De plus, étant donné la définition de sens commun de la sécurité privée et celle qui est donnée implicitement par la législation qui se réfère à ce type de sécurité, il semble que les fonctionnaires publics qui partagent avec la police le statut d'agent de la paix, tels que les gardiens de prison, les maires, les gardes-chasse et les douaniers (Freedman et Stenning, 1977, p. 18) doivent également être exclus de la catégorie des occupations de sécurité, si l'on tient à faire de la sécurité privéé une sous-catégorie distincte.

À partir de ce raisonnement, les personnes au service de la sécurité privée peuvent être définies comme étant des personnes employées à des occupations de sécurité et qui sont autres que les membres de la police militaire, de la police publique et des corps de fonctionnaires publics ayant un statut d'agent de la paix. À première vue, cette définition peut sembler inadéquate puisqu'elle caractérise indirectement la police privée comme une catégorie résiduelle. C'està-dire qu'elle précise ce que la sécurité privée n'est pas, sans définir ce qu'elle est. Notons cependant que cet aspect de notre définition, plutôt que de présenter un inconvénient, offre l'avantage d'être conforme à la manière dont on définit la sécurité privée en pratique. Pour la plupart des gens, la sécurité privée est une activité qui est probablement consacrée à la sécurité, sans coïncider avec la police publique ni avec l'appareil militaire.

Cette définition connote également une autre caractéristique de la définition de sens commun de la sécurité privée. Dans la pratique, la ligne de démarcation entre la sécurité privée et les autres forces de sécurité est en quelque sorte floue. Son tracé est flou de deux façons. D'abord, il n'est pas toujours clair quelles sont précisément les personnes qui doivent être rangées dans la catégorie des autres occupations de sécurité. Les préposés à la circulation automobile devraient-ils être ainsi rangés dans la catégorie de la police publique

2. Nous traduisons l'expression "private security person", dont l'emploi est normal en anglais, par «personne au service de la sécurité privée», qui est une périphrase moins familière en français. Traduisant une communication qui relève davantage de la science que de la littérature, nous avons délibérément choisi de sacrifier l'élégance à l'exactitude. Comme on le verra, l'expression "personne au service de la sécurité privée» est fréquemment utilisée dans le texte. (n.d.t.). 
comme le suggèrent Freedman et Stenning (1977, p. 18), bien que leur statut d'agent de la paix soit étroitement limité? D'autre part, la question de savoir quelles sont au juste les autres occupations qui, en plus de la police et de l'armée, devraient être exclues de la catégorie de la sécurité privée, apparaît comme étant autant une affaire de convention qu'une affaire de principe. Il semble ainsi que les gardeschasse pourraient tout autant figurer dans la catégorie de la police privée qu'ailleurs. Ainsi, bien que leurs membres ne bénéficient pas du plein statut d'agent de la paix, la police des chemins de fer du Canadien National, celle des chemins de fer du Canadien Pacifique et la Police des ports disposent tout de même d'un statut limité d'agent de la paix qui les place «sur la ligne de démarcation entre la police publique et la police privée» comme le soulignent Freedman et Stenning (1977, p. 37).

\section{QUELS TYPES DE SÉCURITÉ PRIVÉE EXISTE-T-IL?}

La sécurité privée est généralement classée en fonction de quatre critères: premièrement, selon le lien qui unit l'agence de sécurité à ses clients; deuxièmement, selon que l'agence est licenciée ou non; troisièmement, selon que le travail consiste dans du gardiennage ou de l'enquête et, finalement, selon que les personnes au service de la sécurité privée assument les fonctions de sécurité directement ou indirectement, en tant que servants d'un équipement de sécurité.

\section{a) LES AGENCES DE SÉCURITÉ PAR CONTRAT, PAR OPPOSITION AUX SERVICES DE SÉCURITÉ INTERNES ${ }^{3}$}

Les deux catégories de services de sécurité auxquels on fait appel le plus souvent sont les agences par contrat et les services internes. Les agences par contrat désignent les «agences et agents qui font commerce d'assurer... des services de [de sécurité] aux autres [les clients] en échange d'un salaire ou d'une prime. Les agences et agents par contrat peuvent ainsi être distingués des services [de sécurité]... internes qui assurent leurs services exclusivement à la compagnie [l'institution ou le particulier] qui les emploie sur une base plus ou moins permanente» (Freedman et Stenning, 1977, p. 46).

3. Agences de sécurité internes et agences de sécurité par contrat traduit l'opposition anglaise «in-house security" et "contract security». Cette traduction est conforme à la pratique des rapports et documents de la Commission de réforme du droit du Canada (n.d.t.). 
b) LES AGENCES DE SECCURITÉ PRIVÉE LICENCIÉES PAR OPPOSITION AUX AGENCES NON LICENCIÉES

Au Canada, les agences de sécurité privée ne sont pas toutes soumises à la réglementation de statuts exigeant l'obtention d'une licence. Bien que l'amplitude de la législation concernant l'attribution des licences varie quelque peu d'une province à l'autre et à l'intérieur même d'une province, dans toutes les provinces disposant de statuts sur les licences, la plupart des agences de sécurité par contrat sont licenciées tandis que les services de sécurité internes ne le sont pas. À travers tout le Canada, il existe une sous-catégorie importante d'agences de sécurité par contrat qui n'a pas à être licenciée en vertu de la loi qui régit la sécurité privée; il s'agit des agences de renseignement sur le crédit. Cependant, en Colombie Britannique, au Manitoba, en Nouvelle-Écosse, en Ontario et en Saskaschewan, «en vertu de la loi de la protection du consommateur... il leur est demandé de détenir une licence afin d'opérer légalement» (Freedman et Stenning, 1977, p. 48).

\section{c) LES GARDIENS DE SÉCURITÉ ET LES ENQUÊTEURS}

Dans le domaine de la sécurité, il est de règle de faire une distinction entre les personnes au service de la sécurité privée qui assurent un service de gardiennage et celles qui assurent un service d'enquête. Au Canada, cette distinction a pris de l'importance en étant intégrée à la législation concernant les licences. Ceci a eu pour effet de rendre la distinction plus importante encore pour les agences de sécurité par contrat. La plupart des statuts provinciaux définissent le gardien de sécurité comme une personne qui «agit en tant que gardien ou veilleur, qui garde ou patrouille ou assure d'autres services de sécurité dans le but de protéger les personnes ou la propriété» (Freedman et Stenning, 1977, p. 46).

Bien qu'il y ait moins d'accords au niveau de la législation provinciale en ce qui concerne la définition des enquêteurs privés, il est possible, comme le démontrent Freedman et Stenning, d'élaborer une «définition synthétique». La Loi canadienne sur les licences définit l'enquêteur privé comme étant une personne qui:

1. recherche et fournit des renseignements sur la réputation et les activités des personnes et la nature de l'entreprise ou de l'occupation des personnes (non applicable au Québec);

2. recherche les contrevenants à la loi (non applicable en Alberta ou en Colombie Britannique); 
3. recherche les personnes ou les biens portés disparus (non applicable au Québec ou en Colombie Britannique) (1977, p. 47).

\section{d) L'ÉQUIPEMENT DE SÉCURITÉ}

Dans tous les secteurs de l'activité humaine, le développement technologique implique fréquemment que des tâches autrefois accomplies par des gens peuvent être et sont maintenant souvent accaparées par des machines. Ce phénomène n'est pas nouveau. Les dix-neuvième et vingtième siècles ont toutefois connu des développements technologiques énormes, qui ont entraîné de sérieuses conséquences sociales. Les développements en technologie électronique en particulier, ont eu un profond effet sur la sécurité. Avant les progrès rendus possibles par la technologie électronique, les méthodes mécaniques de protection avaient peu évolué depuis des temps immémoriaux. Alors que nous avons, par exemple, inventé et perfectionné de nouvelles serrures plus efficaces, il n'y a comme tel rien de nouveau dans l'usage de dispositifs de verrouillage. La technologie électronique a toutefois modifié de façon radicale la nature de l'équipement de sécurité. Freedman et Stenning constatent qu' «il est effectivement possible d'arguer que l'élément humain est, ou est en voie de devenir, une considération secondaire dans plusieurs secteurs de la sécurité privée» (1977, p. 209).

Les progrès de la technologie électronique dans le champ de la sécurité ont créé une distinction de plus en plus marquée entre les personnes au service de la sécurité privée qui servent un équipement, comme les gens qui répondent au déclenchement d'une alarme, et celles qui opèrent dans une relative indépendance de tels équipements.

\section{QUE FONT LES PERSONNES AU SERVICE DE LA SÉCURITÉ PRIVÉE?}

\section{a) LA PAIX PUBLIQUE ET LA PAIX PRIVÉE ${ }^{4}$}

En proposant une définition de la sécurité privée, nous avons déjà largement fourni l'équivalent d'une délimitation assez précise des activités des personnes au service de la sécurité privée. Elles assurent la protection de l'information, des personnes et des biens. En

4. L'expression «la paix» (the peace) est ici prise dans son sens juridique de quiétude et non dans son acception politique restreinte, qui réfère à l'absence de conflit armé entre les États (n.d.t.). 
faisant cela, elles assurent la préservation de la paix, tout comme la police publique. Ceci n'a rien d'étonnant, étant donné que le concept de la paix est issu d'un souci pour les personnes et ce qui leur appartient. Comme l'a indiqué récemment Keeton, l'ancien concept anglo-saxon de frith duquel découle notre concept de «la paix», désigne «le droit d'inviolabilité inhérent aux personnes, aux lieux et aux collectivités» (1975, p. 3).

Cette analogie entre la fonction de la police privée et celle de la police publique a été soulignée par des commentateurs en GrandeBretagne (voir, par exemple, Wiles et McClintock, 1972), aux ÉtatsUnis (voir, par exemple, Post, 1971) et au Canada (Jeffries, 1977), et elle explique cette préférence que certains auteurs accordent au terme «police privée» lorsqu'ils se réfèrent à la sécurité privée.

Les forces de la sécurité privée font cependant plus que préserver la paix publique. À la différence de la police publique qui n'est pas «légalement responsable de faire respecter les lois et règlements ayant fait l'objet d'une mise en vigueur privée» (Kakalik et Wildhorn, 1972, p. 57), l'application de ces règles constitue une partie intégrante des responsabilités de plusieurs des personnes au service de la sécurité privée. Les responsabilités de la sécurité privée débordent ainsi celles de la police publique et intègrent ce que l'on pourrait appeler, faute d'un meilleur terme, la préservation des «paix privées».

\section{b) LES ACTIVITÉS DE SECCURITE}

Nous passons maintenant à l'analyse plus détaillée des fonctions remplies par les personnes au service de la sécurité privée. Actuellement, la seule recherche publiée sur les activités de la sécurité privée au Canada est une étude de Jeffries (1977) sur les services de sécurité internes en Ontario. Même si cette recherche se limite à l'Ontario et aux services de sécurité internes, et même si l'analyse proposée est d'un niveau élémentaire, elle fournit un point de départ utile pour dresser l'inventaire des fonctions performées par la sécurité privée; les exemples choisis par Jeffries représentent en effet un vaste éventail des usagers des services fournis par la sécurité privée. Les résultats de Jeffries sont basés sur l'analyse de vingt-et-un services de sécurité internes, opérant dans le cadre de sociétés commerciales, industrielles, minières ou dispensant des services personnels. Alors qu'on trouvera sans aucun doute quelques différences dans les activités accomplies par des agences de sécurité à contrat ou privées, dans d'autres parties du Canada, des catégories similaires d'usagers 
sont susceptibles de formuler des demandes similaires à l'endroit des agences de sécurité privée, peu importe où celles-ci opèrent ou la façon dont elles sont organisées. En interprétant les résultats de Jeffries, nous devons toutefois souligner que son échantillonnage comporte une lacune significative, soit l'absence d'organismes gouvernementaux de sécurité privée. Ayant cette limitation présente à l'esprit, nous procéderons à l'examen de ses résultats.

Jeffries décrit les fonctions exercées par des personnes occupant différents postes au sein d'organismes de sécurité internes. $\mathrm{Si}$ ses résultats sont classés selon que les activités accomplies correspondent surtout à des fonctions d'enquête ou de gardiennage, il en découle l'analyse dont nous présenterons maintenant les résultats (1977, p. 15).

Ces résultats viennent confirmer nos conclusions générales concernant la relation entre le travail de la police publique et celui de la sécurité privée. Les personnes au service de la sécurité privée étudiées par Jeffries se chargeaient, comme la police, d'enquêter sur les crimes, de maintenir l'ordre et de répondre aux situations d'urgence et de crise. De plus, ces personnes étaient, contrairement à la police, responsables de l'application des réglementations privées, telles que l'accès à certains lieux.

Fonctions d'enquête

\begin{tabular}{ll}
\hline Postes & Fonction \\
\hline Chargé d'investigations & $\begin{array}{l}\text { Enquête sur des cas particuliers de } \\
\text { fraude, de vol ou sur d'autres illé- } \\
\end{array}$ \\
galités.
\end{tabular}

Enquêter (sur l'étage)

Découvre et appréhende les voleurs à l'étalage, en conformité avec la politique de la compagnie.

Faux client (dans des commer- Effectue des contrôles sur l'«honces de vente au détail) nêteté» des caissiers et des caissières.

Surveillant d'étage

Patrouille les locaux de la compagnie à intervalles irréguliers et imprévus.

Réagit aux situations d'urgence qui se présentent au cours des rondes. 
Fonctions de gardiennage

\begin{tabular}{ll}
\hline Poste & Fonction \\
\hline Gardien factionnaire & Affecté à un «poste de contrôle» \\
& sur la propriété de la compagnie. \\
& Contrôle l'entrée et la sortie de \\
& zones spécifiques.
\end{tabular}

Répond aux situations d'urgence en cas de nécessité.

Patrouille munie d'une horloge à poinçon

Veilleur de nuit
Effectue des rondes de routine selon le temps enregistré par l'horloge à poinçon.

Responsable de la sécurité matérielle durant les heures d'inactivité.

Les résultats de Jeffries servent, en plus, à mettre en évidence une autre différence entre la police et la sécurité privée. Bien que la sécurité privée s'occupe à la fois de l'application des lois et des réglementations privées, elle limite généralement ses interventions (au périmètre) de la propriété privée de ses clients. La sécurité privée a un accès de routine régulier à la propriété privée, alors que la police publique ne s'introduit généralement sur la propriété privée que lorsqu'elle y est spécifiquement invitée. Ceci signifie qu'en ce qui concerne les zones sous sa responsabilité, la sécurité privée n'est généralement pas assujettie à une restriction majeure imposée à la police publique, soit l'accès limité à des lieux privés. Les personnes au service de la securité privée invoquent souvent cette différence à l'appui de l'argument selon lequel elles seraient dans une position beaucoup plus propice à la prévention des crimes que la police, dont la tâche est généralement réduite à réagir au crime après sa perpétration.

Cette discussion sur les activités de la sécurité privée soulève la question de savoir quels intérêts elle sert, à savoir pour qui ceux qu'elle emploie travaillent-ils? Théoriquement, la police publique opère au titre de serviteur de la population tandis que les personnes au service de la sécurité privée agissent clairement dans l'intérêt d'individus ou d'organismes particuliers. Même si ces personnes n'abandonnent pas pour autant leurs responsabilités de citoyens, elles reçoivent néanmoins leurs instructions, en tant que personnes au service de la sécurité privée, des employeurs dont elles sont chargées de 
protéger les intérêts. Ainsi, quand nous sommes policés par la police publique, nous le sommes théoriquement dans notre propre intérêt. Et quand nous sommes policés par la sécurité privée, toute communauté d'intérêts n'est, autant en théorie qu'en pratique, qu'un aspect fortuit de cette relation; nous sommes policés, à l'intérieur du cadre de la protection que nous apporte la loi, dans l'intérêt de celui qui emploie la personne au service de la sécurité privées.

\section{QUELLES SONT LES ORIGINES HISTORIQUES DE LA SÉCURITÉ PRIVÉE?}

Pour comprendre le rapport qui existe entre la police publique et la sécurité privée au niveau de leurs responsabilités, il est utile d'examiner brièvement les racines historiques de chacun de ces deux types de police.

Le concept de «la paix» qui forme le centre de notre notion de sécurité emprunte ses origines, comme nous l'avons proposé, à l'ancien concept anglo-saxon de Frith. Comme nous l'avons vu, ce concept faisait référence à la paix dans un sens très localisé, en insistant sur l'inviolabilité des personnes, des lieux et des collectivités. Le concept moderne de la paix publique représente une extension et un remaniement de cette idée primitive.

La première étape dans cette évolution consista à définir la paix du roi comme une paix d'un ordre particulier. Le «frith» ou "grith" du roi, comme on appelait ce droit, concernait non seulement la personne du roi mais s'étendait à toute sa Maison. L'extension progressive de cette notion permit d'y englober l'ensemble du royaume. Selon l'expression de Maitland (1913, p. 108), la paix du roi «dévore toutes les autres paix». Elle s'égale à la paix publique et le souverain devient responsable d'assurer non seulement la paix de sa Maison mais la paix du royaume.

L'extension de la paix du roi a eu pour effet de reléguer dans l'ombre le concept originel de «frith», de telle sorte qu'il nous semble maintenant étrange de parler de paix privées. Ce concept est pourtant enchâssé dans d'autres notions plus familières; par exemple, dans la distinction entre le droit civil et le droit criminel et entre un préjudice privé et un préjudice public. L'ancien concept de frith constitue le fondement historique de la paix que préserve la sécurité

5. Là où la sécurité privée travaille pour des gouvernements, sa position vis-àvis des intérêts qu'elle sert ressemble à celle de police publique. 
privée, de la loyauté de cette dernière à des employeurs particuliers et de l'étroitesse géographique du foyer de ses responsabilités.

À l'origine, les représentants de la collectivité responsables du maintien du «frith» étaient investis de leur autorité directement par la collectivité locale ou le groupe de parenté, dont ils étaient les mandataires (Critchley, 1967). Avec l'émergence du concept de la paix publique, ces mandataires de la collectivité se trouvèrent dans une position délicate et ambiguë, puisqu'ils étaient en même temps responsables devant deux autorités, le souverain et leur collectivité locale. Au cours des siècles, cette tension a été systématiquement réduite de sorte qu'aujourd'hui, la police, tout en étant organisée en unités locales, assume des responsabilités qui se limitent à la préservation de la paix publique et ses membres, en tant qu'agents de la paix, exercent une autorité qui émane directement de l'autorité souveraine (Critchley, 1967).

La tension provoquée par une double imputabilité persiste cependant dans le cas de la personne au service de la sécurité privée, dont la position est comparable à plusieurs égards à celle de l'ancien hundredsman ${ }^{6}$ responsable de maintenir le «frith» dans une collectivité ou un groupe de parenté, tout en devant respecter ses obligations envers le roi. En tant que citoyen, la personne au service de la sécurité privée a des responsabilités envers l'autorité souveraine; mais en tant que personne au service de la sécurité privée, comme telle, elle est responsable devant son employeur. Contrairement au hundredsman toutefois, cette personne ne remplit pas souvent de fonctions publiques, sauf si elle est assermentée comme constable spécial. C'est essentiellement à son employeur qu'elle accorde sa loyauté.

\section{QUELS SONT LES POUVOIRS LÉGAUX, LES DROITS ET LES DEVOIRS DES PERSONNES AU SERVICE DE LA SÉCURITÉ PRIVÉE?}

Comme nous l'avons laissé entendre au cours de notre discussion sur les origines historiques des personnes au service de la sécurité privée, celles-ci n'ont habituellement que les pouvoirs, droits et devoirs des simples citoyens ou les pouvoirs qui leur sont délégués par leur employeur, ce dernier étant, dans la plupart des cas, «le pro-

6. Cette expression est intraduisible en français. Elle désigne celui qui exerçait l'autorité légale dans une juridiction primitive crée par le droit anglais. Le terme de hundred apparaît dans des statuts britanniques qui remontent au $\mathrm{x}^{\mathrm{e}}$ siècle (les lois du roi Edmond I, 939-946) et référait à l'origine à un lopin de terre suffisant pour nourrir une famille de paysans (n.d.t.). 
priétaire ou une personne en possession légitime de biens» (Code criminel canadien, art. 449(2)).

Notre législation accorde certains pouvoirs, certains devoirs et certains droits aux simples citoyens et aux propriétaires de biens. En tant qu'agents de ces propriétaires et simples citoyens, les personnes au service de la sécurité privée exercent ces pouvoirs, ces devoirs et ces droits. Si nous voulons comprendre le statut légal des personnes au service de la sécurité privée, nous devons, par conséquent, comprendre la situation des simples citoyens et des propriétaires de biens. En ce qui concerne le maintien de l'ordre public, le Juge Morand (1976) a récemment affirmé que les simples citoyens ont une obligation de droit commun de maintenir la paix publique. Par contraste, Freedman et Stenning (1977, p. 259-260) ont soutenu que la situation des simples citoyens n'est pas du tout claire, au Canada. C'est avec beaucoup d'ambivalence, soulignent-ils, que la loi canadienne stipule aux citoyens la nature de leur implication dans le maintien de la paix publique. Ils arrivent à la conclusion que si le public a clairement le droit de maintenir la paix publique, il est douteux qu'il en ait le devoir.

Pour l'exercice de ce droit au maintien de la paix publique, la loi canadienne accorde aux citoyens certains pouvoirs légaux en rapport avec des actes tels que l'usage de la force, les fouilles, les saisies et l'arrestation. Dans le cadre de cet article, il n'est pas possible d'envisager en détail les limites de ces pouvoirs ${ }^{7}$. Nous considérons plutôt, à titre d'illustration, les pouvoirs qui autorisent les citoyens à poser l'un de ces actes légaux, en l'occurrence l'arrestation.

Le Code criminel canadien constitue la principale source d'autorité pour ce qui est des pouvoirs du citoyen d'effectuer une arrestation. Comme nous l'avons mentionné, le Code établit une distinction entre d'une part les simples citoyens et, d'autre part, les propriétaires, les personnes en possession légitime de biens et leurs agents. Le Code limite les pouvoirs d'arrestation de ces deux catégories de simples citoyens aux cas d'arrestation sans mandat. Dans le cadre de cette restriction générale, les pouvoirs d'arrestation conférés aux simples citoyens sont définis par l'article 449 , comme suit:

1. Toute personne peut arrêter sans mandat

a) un individu qu'elle trouve en train de commettre un acte criminel, ou 
b) un individu qui, d'après ce qu'elle croit pour des motifs raisonnables et probables, i) a commis une infraction criminelle, et ii) est en train de fuir des personnes légalement autorisées à l'arrêter et est immédiatement poursuivi par de telles personnes.

Comme le précise cet article, dans des circonstances normales, un citoyen ne peut arrêter quelqu' un que pour un acte criminel; c'està-dire, pour des infractions graves telles que le viol, l'incendie criminel, le vol qualifié, le vol avec effraction et ainsi de suite. De plus, dans des circonstances normales, il ne peut procéder à une arrestation pour l'une de ces infractions que s'il trouve la personne en train de commettre l'infraction. Ceci signifie qu'un citoyen ne peut normalement arrêter une personne simplement parce qu'il croit, ou même «sait", que celle-ci a commis un acte criminel.

Le Code criminel prévoit une légère augmentation des pouvoirs d'arrestation des simples citoyens lorsque ceux-ci sont amenés à assister quelqu'un disposant de l'autorité légale de procéder à une arrestation, pourvu que cette dernière personne soit engagée dans la «poursuite active» de l'individu à arrêter. Dans ces circonstances, le citoyen peut procéder à une arrestation non seulement pour des actes criminels mais aussi pour des infractions criminelles ${ }^{8}$. On comprendra que dans ces circonstances, le Code n'exige pas que la personne arrêtée par un citoyen prêtant assistance soit surprise en train de commettre une infraction, mais simplement que le citoyen en question ait des «motifs raisonnables et probables» de croire que la personne qu'il seconde possède l'autorité légale pour effectuer une arrestation.

Ces pouvoirs d'arrestation conférés au citoyen augmentent lorsque ce citoyen est un propriétaire, une personne en possession légitime de biens ou l'agent d'une telle personne?. Dans ces circonstances, une personne peut arrêter quiconque est «trouvé en train de

8. En anglais: indictable offenses, que nous traduisons par «acte criminel» et criminal offense par «infraction criminelle», pour faire court. Au sens strict l'expression «criminal offense» devrait être traduite par la longue expression «infraction punissable sur déclaration sommaire de culpabilité». La différence entre ces deux catégories d'infractions est largement une question de "gravité", les premières étant plus graves que les secondes, (n.d.t.).

9. Il s'agit de l'article 449(2) du Code criminel. Cet article prévoit que: 2. Quiconque est a) la propriétaire ou une personne en possession légitime d'un bien, ou b) une personne autorisée par le propriétaire ou par une personne en possession légitime d'un bjen, peut arrêter sans mandat une personne qu'il trouve en train de commettre une infraction criminelle sur ou concernant ledit bien. 
commettre ${ }^{10} \gg$ une infraction criminelle (par opposition à un acte criminel), à condition que cette infraction soit commise «sur ou par rapport à» la propriété en question. Cet article accorde aux propriétaires, à ceux qui sont en possession légitime de biens et à leurs agents, des pouvoirs d'arrestation plus étendus que ceux des autres citoyens en étendant le champ de leurs pouvoirs d'arrestation, au-delà du cadre des seuls actes criminels, à toutes les infractions au Code criminel, même en l'absence de présomption quant au déroulement d'une «poursuite active».

Cet élargissement des pouvoirs d'arrestation reflète la place centrale qu'occupe l'institution de la propriété privée dans notre société et témoigne du fait que notre législation établit des droits de propriété et fournit aux propriétaires et aux personnes en possession légitime de biens des moyens coercitifs soutenus par l'État pour s'assurer de ces droits et pour les défendre (Chambliss et Seidman, 1971; Freedman et Stenning, 1977).

D'après les dispositions que nous venons d'examiner, il est clair que les pouvoirs légaux d'arrestation dont disposent la plupart des personnes au service de la sécurité privée sont considérablement moins étendus que ceux de la police publique qui, dans les cas d'actes criminels, n'est généralement pas limitée par la clause du «trouve en train de commettre» mais qui peut procéder à une arrestation pourvu qu'elle ait des motifs raisonnables et probables de croire qu'un crime a été ou est sur le point d'être commis (Code criminel canadien, art. $480(1))$. Les effets pratiques de cette différence sont toutefois loin d'être aussi considérables qu'on pourrait le soupçonner. Par contraste avec la police publique, les personnes au service de la sécurité privée, dans leur qualité d'agents des propriétaires ou de ceux qui sont en possession légitime de biens, ont fréquemment un accès de routine à des lieux privés où des crimes contre la propriété sont susceptibles d'être commis. Ceci signifie que ces personnes sont plus susceptibles que la police publique de surprendre un individu en train de commettre une infraction criminelle et qu'elles ont vraisemblablement moins besoin qu'elle de pouvoir procéder à une arrestation à partir de soupçons. Dans le cas où la personne au service de la sécu-

10. Les tribunaux canadiens ont interprété l'expression «trouve en train de commettre» comme signifiant «trouve apparemment en train de commettre». Il en résulte qu'une personne ne sera pas trouvée coupable d'avoir procédé à une arrestation illégale même s'il était prouvé par la suite que la personne arrêtée n'était pas effectivement en train de commetre l'infraction en question; il suffit qu'on réussisse à faire valoir que la personne arrêtée était apparemment en train de commettre l'infraction. Pour une discussion sur cette distinction, voir Freedman et Stenning (1977, p. 79-82). 
rité privée ne surprend pas une personne en train de commetre une infraction criminelle mais la trouve sur les lieux de la propriété qu'elle protège, il est significatif de constater qu'elle peut en général toujours arrêter cette personne, si elle souhaite la détenir, en vertu des dispositions formulées dans les lois provinciales sur l'intrusion (provincial Tresspass Acts); ces dispositions reconnaissent, comme le soulignent Freedman et Stenning, «des motifs d'arrestation même lorsque aucune infraction criminelle n'a été commise» (1977, p. 110). Ainsi, dans les cas où une personne est soupçonnée d'avoir commis une infraction criminelle sans avoir été prise sur le fait, les personnes au service de la sécurité privée ont peu de difficulté à détenir légalement cette personne pour permettre une enquête ultérieure, si elles le désirent. Il est aussi significatif de noter que dans les cas où aucune urgence ne nécessite la détention, les personnes au service de la sécurité privée peuvent, en pratique, se prévaloir des pouvoirs d'arrestation les plus étendus, en communiquant simplement avec la police et en lui persuadant qu'il existe des motifs raisonnables et probables de soupçonner qu'un acte criminel a été commis.

À la lumière de ces considérations, il est évident que là où elles protègent la propriété, les personnes au service de la sécurité privée jouissent, en pratique, sensiblement de la même position que les policiers en ce qui concerne le recours à l'arrestation, comme étant le moyen de résoudre les problèmes qu'elles rencontrent.

Non seulement les personnes au service de la sécurité privée disposent-elles en pratique de pouvoirs d'arrestation qui sont sensiblement les mêmes que ceux de la police publique mais dans certaines circonstances, certaines d'entre elles ont des pouvoirs légaux identiques à ceux de la police publique par suite de leur accession au statut d'agent de la paix, à travers une nomination comme constable spécial. Quand des personnes au service de la sécurité privée sont nommées constables spéciaux, elles deviennent des quasi-policiers, en vertu du statut d'agent de la paix que leur confère cette nomination ${ }^{11}$. Dans les cas où une personne au service de la sécurité privée est nommée constable spécial, avec un statut d'agent de la paix dont la seule limitation tient dans une contrainte géographique (par exem-

11. Ce statut est de nature quasi policière, car ces nominations accordent rarement aux personnes ainsi nommées le plein statut d'agent de la paix, mais limitent leurs pouvoirs à des zones spécifiques et/ou les limitent à des périodes de temps spécifiques. Par exemple, une personne au service de la sécurité privée peut être nommée constable spécial pour appliquer une réglementation municipale sur le stationnement à l'intérieur et autour du site d'une exposition, pour la durée de cette exposition. 
ple, une «police» de campus), elle dispose, par rapport à cette zone, des mêmes pouvoirs légaux qu'un agent de police attitré. Toutefois, son accès privilégié à la propriété privée signifie qu'à l'intérieur du territoire relevant de sa juridiction, elle n'est pas soumise aux mêmes contraintes qui sont imposées aux policiers en titre par les institutions qui veillent au respect de notre vie privée (Stinchcombe, 1963).

Là où des personnes au service de la sécurité privée sont nommées constables spéciaux, la difficulté conflictuelle de porter deux chapeaux, à laquelle nous avons fait allusion précédemment, n'en est que plus aiguë. Dans ces circonstances, leur situation correspond exactement à celle des hundredsman anglo-saxons. Elles sont, d'une part, des représentants de la Couronne qui reçoivent autorité et directives uniquement de la loi. D'autre part cependant, en tant qu'employés agissant au nom de leurs employeurs, c'est de ces derniers qu'elles font dériver leur autorité. Théoriquement, cette double autorité devrait servir à réduire le contrôle exercé par les employeurs sur les personnes au service de la sécurité privée, ces dernières devenant en vertu de leur statut d'agent de la paix des représentants indépendants de la Couronne. En pratique toutefois, cette éventualité est peu probable, puisque ce sont les exigences de leur employeur qu'elles doivent satisfaire, si elles veulent garder leur emploi ${ }^{12}$.

Il y a, outre ces considérations, un aspect additionnel des circonstances pratiques affectant le travail des personnes au service de la sécurité privée qui doit être examiné, si nous voulons pleinement évaluer l'absence relative de signification de la différence entre les pouvoirs de la police et ceux de la sécurité privée. C'est la question du consentement.

En fixant les limites de ce que les gens peuvent se faire les uns aux autres, notre législation intervient, dans la plupart des cas, pour protéger les personnes contre l'imposition de contraintes exercées contre leur volonté. La plupart de nos lois (à l'exception de certaines infractions contre les mœurs) sont relativement indifférentes à l'égard de ce que se font mutuellement les gens, à la condition qu'ils consentent à ce qui se passe. Ce principe légal est d'une grande importance pour le rapport qui s'établit entre les forces de sécurité et ceux qu'elles surveillent. En ce qui a trait aux limites légales que nous venons de considérer, «toute la situation est modifiée, s'il peut être démontré que le citoyen a consenti à de telles intrusions (par exem-

12. Pour un examen approfondi des nominations de constables spéciaux et de leur usage au Canada, voir Stenning et Cornish (1975, p. 196-212). 
ple, s'est volontairement soumis à une détention ou une fouille). Le policier ou l'agent de sécurité privée n'ont plus à s'appuyer sur les divers pouvoirs qui leur sont conférés par le Code criminel et par d'autres statuts; en effet, le consentement de la personne fouillée, détenue, interrogée, etc., suffit à rendre l'intrusion légalement justifiable dans presque tous les cas» (Freedman et Stenning, 1977, p. 68). Par conséquent, «l'agent de sécurité privée qui s'efforce prudemment d'obtenir un consentement valide des personnes avec lesquelles il entre en relation n'a guère à se préoccuper des diverses lois qui ont été promulguées ou qui ont été (par la suite) explicitées par les tribunaux en vue de protéger ces personnes contre des intrusions ou des contraintes illégales» (Freedman et Stenning, 1977, p. 76).

Le personnel de sécurité n'a généralement pas de difficulté à obtenir un consentement. En effet, très souvent, le consentement suit de l'implication d'une personne dans une situation donnée. Il se peut, par exemple, que l'une des conditions à l'obtention d'un emploi soit que l'employé consente à être fouillé au moment de quitter son lieu de travail. De façon similaire, une ligne aérienne peut exiger de ceux qui montent à bord de ses avions qu'ils consentent à être fouillés. Quelle que soit la raison qu'ils allèguent, il est clair que les personnes au service de la sécurité privée sont souvent capables d'obtenir d'une manière relativement facile le consentement requis pour ce qu'ils désirent faire. Ce fait atténue davantage l'impact réel de la différence entre les pouvoirs légaux de la police publique et ceux de la sécurité privée. On pourrait en effet arguer qu'une personne au service de la sécurité privée se trouve probablement dans une «meilleure» position que la plupart des policiers, étant plus à même d'amener les gens à consentir à son intervention.

En résumé, il semble qu'en pratique, les personnes au service de la sécurité privée soient non seulement fréquemment en mesure d'exercer un contrôle sur une plus grande part de notre existence que ne le fait la police publique puisque leurs responsabilités débordent le cadre de la paix publique pour s'étendre jusqu'aux "paix privées", mais il semble de plus que leurs aptitudes à interférer en fait avec l'exercice de notre liberté soient aussi et peut-être même plus étendues que celles de la police publique.

\section{COMMENT LA SÉCURITÉ PRIVÉE EST-ELLE RÉGLEMENTÉE AU CANADA?}

Stenning et Cornish (1975) ont attentivement analysé la question de la réglementation de la sécurité privée. Plus récemment, 
Freedman et Stenning (1977) ont résumé les grandes lignes de la réglementation de la sécurité privée au Canada et c'est vers leur compte rendu que nous nous tournerons, encore une fois.

Ils précisent que légalement, l'attribution de licences implique que les personnes et les organismes à qui on impose cette formalité ne peuvent assurer des services de sécurité sans avoir d'abord obtenu cette licence. Il est significatif de constater «qu'en dehors du droit de faire légalement des affaires... de telles licences ne confèrent directement aucun autre statut légal ou pouvoirs à leurs détenteurs", bien que «le fait d'avoir été officiellement licencié pour accomplir certaines fonctions... puisse... leur offrir indirectement une plus grande protection en cas de responsabilité criminelle ou civile (par exemple, une «excuse légale»), en vertu de certaines dispositions statutaires» (1977, p. 47).

Au Canada, toutes les agences de sécurité privée ne sont pas tenues d'être licenciées. Dans la suite de cette partie, nous distinguerons diverses catégories d'effectifs de sécurité et nous indiquerons, à l'intérieur de chaque catégorie, quelles personnes et quels organismes ont besoin d'une licence.

\section{a) LES AGENTS ET AGENCES DE GARDIENS DE SÉCURITÉ À CONTRAT}

Freedman et Stenning écrivent que «l'Alberta, la Colombie Britannique, le Manitoba, l'Ontario, le Québec, le NouveauBrunswick et la Nouvelle-Écosse ont tous une législation en vigueur qui exige que les agents et agences de gardiens de sécurité soient of ficiellement licenciés afin de faire légalement des affaires» (1977, p. 45-46). Depuis lors, une législation semblable est entrée en vigueur à Terre-Neuve et est en train d'être rédigée dans l'île-du-PrinceÉdouard. Comme le laissent supposer Freedman et Stenning (1977), dans le passage que nous venons de citer, la Saskatchewan exclut les gardiens de sécurité à contrat du cadre de sa législation. Bien que ce soit la seule province à exclure les gardiens de sécurité, toutes les législations provinciales excluent d'une manière explicite certaines catégories de sécurité à contrat. En identifiant les personnes et les organismes exclus, Freedman et Stenning notent:

Nous pouvons classer dans cette catégorie les inventeurs, les fabricants, les distributeurs, les installateurs et le personnel de service pour toutes sortes d'articles de "quincaillerie» (hardware) de sécurité, y compris les serrures, coffres-forts, systèmes d'alarme, dispositifs de sûreté, panneaux et vêtements à l'épreuve des balles et du feu, chiens dressés, armements de 
divers types autres que des «armes à autorisation restreinte», systèmes de contrôle des entrées, systèmes de contrôle des marchandises et équipement de surveillance et d'écoute électroniques. Sont aussi compris dans cette catégorie les opérateurs de détecteurs de mensonge commerciaux (polygraphistes, opérateurs d'évaluateur de stress psychologique), analystes de voix (opérateurs de spectrographe) et, autres experts scientifiques (experts en dactyloscopie ou experts en écriture, en balistique, etc.), psychologues industriels et spécialisés dans les problèmes de sécurité et autres «Consultants en services sécuritaires» et en administration qui, bien qu'étant essentiellement spécialisés en matière de sécurité, ne fournissent pas le genre de services de sécurité requérant une licence en vertu des divers règlements d'attribution de licences au niveau provincial. Certains services de courrier peuvent également opérer sans licence et le personnel chargé de l'écoute et de la réponse aux signaux d'alarme (ce personnel n'étant pas nécessairement le même que celui chargé de l'installation et de l'entretien) se trouvera généralement dans la catégorie du personnel de sécurité contractuel libre (1977, p. 50-51).

Ailleurs, Freedman et Stenning signalent qu'en plus, d'autres groupes font également exception à la législation sur les licences; ceux-ci comprennent la police des chemins de fer et le Corps Canadien des Commissaires.

\section{b) LES AGENCES D'ENQUÊTE PRIVÉES ET LEURS AGENTS}

«Toutes les provinces à l'exception de Terre-Neuve et de l'îledu-Prince-Édouard... exigent que les agences d'enquête privées à contrat, ainsi que les enquêteurs privés, soient détenteurs d'une licence pour opérer légalement» (Freedman et Stenning, 1977, p. 47). Depuis 1977, une telle législation est entrée en vigueur à TerreNeuve et est en train d'être rédigée dans l'île-du-Prince-Édouard. Cependant, dans toutes les provinces où existe une législation, diverses catégories de personnel de sécurité sont, encore une fois, explicitement exclues des statuts sur l'obtention d'une licence.

En plus de l'exemption obvie de la police publique, les règlements exemptent: «la police des chemins de fer, les avocats et les conseillers juridiques ainsi que leurs employés, les enquêteurs chargés de l'estimation de la solvabilité d'une personne et de sa convenance avec un type d'emploi, les enquêteurs et ajusteurs d'assurance, certains employés du gouvernement et des personnes licenciées en d'autres juridictions et temporairement affectées dans la province concernée» (Freedman et Stenning, 1977, p. 48). 
c) LES AGENCES ET LES AGENTS DE RENSEIGNEMENT SUR LE CRÉDIT, Á CONTRAT

Bien que les agences de renseignements sur le crédit et leurs agents ne sont pas soumis aux règlements d'obtention d'une licence concernant les enquêteurs et les gardiens de sécurité à contrat, ils sont obligés dans certaines provinces d'être licenciés en vertu des dispositions d'autres statuts. Cinq provinces (la Colombie Britannique, le Manitoba, la Nouvelle-Écosse, l'Ontario et la Saskatchewan) exigent que ces personnes et ces organismes détiennent une licence en vertu de la loi de la protection du consommateur. Ces statuts «limitent la recherche et la divulgation de renseignements aux seules fins de crédit, de location, d'emploi ou d'assurance. Ils limitent également le genre d'informations susceptibles d'être recueillies et exigent généralement que la personne faisant l'objet d'une enquête soit consentante ou qu'elle soit au moins prévenue qu'une telle enquête est ou a été effectuée. Il est également prévu que la personne puisse avoir accès aux renseignements ainsi recueillis à son sujet» (Freedman et Stenning, 1977, p. 48-49).

\section{d) LES COMMERÇANTS EN ARMES À AUTORISATION RESTREINTE}

En ce qui concerne les commerçants en armes à autorisation restreinte, leur position est exposée par Freedman et Stenning comme suit:

Les personnes qui exercent le commerce de la vente au détail, de la réparation ou qui prennent en gage des armes désignées comme "armes à autorisation restreinte», selon les termes de l'art. 82 du Code criminel sont tenues, en vertu de l'art. 96(2) du Code d'être détenteurs d'un permis à cet effet délivré par le Commissaire de la GRC, un Procureur Général provincial, ou une personne dûment autorisée par l'un d'entre eux (art. 97(1) et (4)). L'article 96(1) du Code oblige également ces personnes à tenir certains registres des transactions impliquant des armes à autorisation restreinte, à produire, à la demande d'un agent de la paix, ces registres pour vérification et enfin à en poster une copie au Commissaire de la GRC s'il en fait la demande par écrit. Les personnes qui fabriquent, achètent, vendent en gros ou importent des armes individuelles sont également soumises à ces obligations de tenir des registres mais elles n'ont pas à détenir de permis pour opérer légalement (1977, p. 49-50).

\section{e) LE PERSONNEL DE LA SÉCURITÉ INTERNE}

Il n'est pas nécessaire actuellement de détenir une licence pour assurer des services de sécurité interne au Canada, même si ce genre de personnel accomplit des fonctions semblables à celles qui sont 
confiées aux employés des agences de sécurité à contrat et même si, du point de vue des personnes soumises à la surveillance et au contrôle, la distinction à contrat/interne est peut-être sans importance.

Le fait que les législateurs réservent un traitement différent à la sécurité à contrat et à la sécurité interne semble suggérer qu'ils sont moins préoccupés par la protection du public contre les activités de la sécurité privée que par celle des employeurs des forces de sécurité à contrat; ces derniers ne pourraient apparemment se protéger de manière satisfaisante faute de contrôler le processus de sélection du personnel de cette sécurité à contrat. C'est ce que laissa entendre en 1973 Michael Warren, alors substitut du solliciteur général pour la province de l'Ontario, au cours de son allocution dans le cadre d'un atelier sur la police et la sécurité privées au Canada. Il constata que les tenants de la position favorable à l'exclusion de la sécurité interne des formalités d'obtention des licences, «font valoir l'argument que les intérêts personnels de l'employeur d'un tel type de personnel l'incitent à porter une attention particulière à la sélection d'employés dignes de confiance et compétents, ainsi qu'à la supervision de leur travail» (1973, p. 56). Dans ses remarques, M. Warren contesta la validité de cette approche. Il laissa entendre que cette distinction entre sécurité à contrat et sécurité interne pouvait ne pas être fondée et que la législation concernant les licences devrait peut-être d'abord se préoccuper des citoyens, dont la vie est affectée par les activités de la sécurité privée. $\mathrm{M}$. Warren présenta ses arguments comme suit:

Il ne fait aucun doute qu'au cours de l'exercice de leurs fonctions, le gardien de sécurité interne, comme l'enquêteur, sont souvent confrontés à des situations susceptibles de porter atteinte à la vie privée et à la liberté d'un individu.

Le recours à l'arrestation, aux fouilles et aux interrogatoires par le personnel de sécurité interne accentue le problème...

Dans leur rapport intitule «Private Police in the United States", Wildhorn et Kakalik laissent entendre que les employeurs «à contrat» défendent des intérêts personnels semblables à ceux des employeurs «internes» en ce qui concerne le choix et la supervision du personnel et que sur tous les points, les motifs invoqués pour réglementer le personnel de sécurité et d'investigation «à contrat» s'appliquent aussi aux agences et au personnel «interne».

Il apparaît clairement qu'il y a lieu de remettre en question l'exclusion du personnel «interne» de notre système de législation $(1973$, p. 56-57). 
À la lumière de cette argumentation, il sera intéressant de voir si la sécurité interne sera incluse dans la nouvelle législation sur les licences, actuellement en préparation en Colombie Britannique et en Ontario.

\section{QUI SONT LES PERSONNES AU SERVICE DE LA SÉCURITÉ PRIVÉE?}

Après avoir considéré le statut légal et la réglementation qui affectent les personnes au service de la sécurité privée, la question qui se pose est la suivante: qui sont les gens qui occupent cette position?

\section{a) LES CADRES DE LA SÉCURITÉ PRIVÉE}

À l'heure actuelle, il y a relativement peu d'information disponible sur les personnes-cadres de la sécurité privée au Canada. Quelques renseignements furent recueillis à ce sujet par Fern Jeffries (1977) au cours d'une étude effectuée en 1974 et 1975 sur la sécurité interne.

Parmi l'échantillon de Jeffries figuraient vingt-quatre personnes qu'elle classa comme administrateurs de sécurité. Ce groupe était essentiellement masculin et présentait une moyenne d'âge de quarante-trois ans. La plupart de ces administrateurs avaient acquis quelque instruction au niveau secondaire. De plus, la moitié d'entre eux avaient fréquenté l'université. Environ un tiers d'entre eux avaient fréquenté un collège communautaire (community college).

En étudiant les antécédents de ces administrateurs de la sécurité, Jeffries constate que le tiers d'entre eux bénéficiant d'une expérience de travail antérieure avaient travaillé en tant que policier public. Quant au salaire, les administrateurs de sécurité interne gagnaient sensiblement plus que leurs employés, 45 pour cent d'entre eux gagnant plus de $20000 \$$ par année. Seulement 5 pour cent des superviseurs de sécurité gagnaient plus de 18000 \$ par année.

\section{b) LES GARDIENS DE SÉCURITÉ}

En ce qui concerne les gardiens de sécurité et les enquêteurs privés, nous sommes en mesure de fournir un profil national. Pour le faire, nous nous appuyons sur le rapport de Farnell et Shearing (1977). Avant de procéder à l'examen de leurs résultats, nous devons toutefois attirer l'attention sur les réserves qu'ils émettent à l'endroit de leur travail, à savoir qu'ils ont éprouvé des difficultés considéra- 
bles à extraire des données satisfaisantes sur le personnel de la sécurité privée des données de recensement disponibles et que leur analyse dépend de la justesse des hypothèses sous-jacentes aux méthodes qu'ils sont utilisées.

En 1971, les gardiens de sécurité au Canada apparaissaient tout à fait comparables aux gardiens de sécurité des États-Unis. À l'instar de Kakalik et Wildhorn (1972, vol. 1, p. 30), Farnell et Shearing rapportent que les gardiens de sécurité sont en moyenne des hommes vieillissants, à la fois mal payés et peu instruits. Ils constatent qu'au Canada, seulement 7 pour cent des gardiens de sécurité étaient des femmes; que 41 pour cent de ceux-ci étaient âgés de cinquante-cinq ans ou plus et qu'ils avaient tendance à gagner moins que le salaire moyen national de 5133 \$, leur salaire annuel moyen étant de 4707 \$. Compte tenu de ce niveau de salaire, il est significatif de constater que les gardiens de sécurité avaient tendance à travailler pendant de longues heures, 14 pour cent travaillant plus de cinquante heures par semaine et 78 pour cent, plus de quarante heures. Quant au degré d'instruction, Farnell et Shearing signalent que jusquà $\mathbf{4 6}$ pour cent des gardes de sécurité n'ont pas complété une neuvième année. Un coup d'œil rapide nous laisse penser que ce profil est encore aussi représentatif aujourd'hui qu'en 1971. Une exception s'applique cependant de toute évidence à plusieurs des grands centres du Canada. Farnell et Shearing rapportent qu'en 1971, plus de 80 pour cent des gardiens de sécurité étaient natifs du Canada. Depuis lors, il semble y avoir eu, en particulier dans les grandes villes, un afflux d'immigrants aux postes de gardiens de sécurité à la fois dans les secteurs de la sécurité à contrat et interne.

\section{c) LES ENQUETEURS PRIVÉS}

Contrairement aux gardiens de sécurité, les enquêteurs privés ont tendance à être en moyenne plus jeunes, plus instruits et mieux payés. De plus, on comptait un pourcentage plus élevé de femmes parmi les enquêteurs privés que chez les gardiens de sécurité, bien que ce nombre demeurait faible (16\%). Dix-sept pour cent étaient âgés de plus de cinquante-cinq ans, ils gagnaient un salaire annuel moyen de 6520 \$ et étaient, dans l'ensemble, mieux instruits que les gardiens de sécurité. Seulement 18 pour cent des investigateurs privés n'avaient pas fréquenté l'école au-delà d'une huitième année.

Comme pour les gardiens de sécurité, les données du recensement de 1971 indiquaient que les enquêteurs privés travaillent pendant des heures relativement longues, 81 pour cent d'entre eux tra- 
vaillant quarante heures par semaine ou plus. La grande majorité $(\mathbf{8 4 \%})$ des enquêteurs privés étaient natifs du Canada. Contrairement aux gardiens de sécurité, il semble que relativement peu d'immigrants soient venus occuper des postes d'enquêteurs privés.

\section{QUELLE EST L'AMPLEUR DE LA SÉCURITÉ PRIVÉE?}

Nous consacrerons cette section à compléter notre description de la sécurité privée au Canada en traitant de la grosseur, de la croissance et du coût de ce service.

À partir de leur analyse des données du recensement, Farnell et Shearing (1977) évaluent à 50200 personnes, le nombre des personnes employées dans des occupations relevant de la sécurité privée au Canada en 1971, en comptant les employés de sécurité du gouvernement dans les établissements correctionnels, etc. La répartition de ce personnel selon le type d'organisation et la province est présentée dans le tableau $n^{\circ} 1$ (Farnell et Shearing, 1977, p. 36).

La répartition de ce personnel en fonction des «divisions industrielles" utilisées par Statistique Canada figure sur le diagramme $n^{\circ} 2$ (Farnell et Shearing, 1977, p. 97).

$\mathrm{Si}$ on ne tient pas compte dans ces chiffres des gardes correctionnels et de ce genre de personnel (ils ne sont habituellement pas considérés comme appartenant à la sécurité privée), Farnell et Shearing évaluent à 36525 le nombre total des personnes exerçant des fonctions de sécurité privée en 1971. Ce chiffre se compare avec celui des effectifs de la police publique rapporté par Statistique Canada et qui était de 39724 pour $1971^{13}$.

Le nombre des personnes employées dans le domaine de la sécurité privée semble avoir augmenté au cours des années soixante. Farnell et Shearing signalent toutefois qu'à cause des changements dans leur présentation, les données de recensement interdisent d'évaluer avec quelque précision le niveau de croissance de la sécurité privée. Ils soutiennent néanmoins que certains calculs suggèrent que durant la période allant de 1961 à 1971, les services de sécurité à con-

13. Ce total ne tient pas compte «des données relatives aux Quartiers généraux de la GRC, les Quartiers généraux divisionnaires et les divisions de formation situées à Ottawa, en Ontario. [Il exclut également] les données relatives aux divers Quartiers généraux de la GRC [en Ontario] et le centre de formation de l'est à Ottawa» (Statistique Canada, Statistiques de l'Administration policière, cat, $n^{\circ} 85-204, p$. 29) cité par Farnell et Shearing (1977, p. 238). 
trat auraient atteint un taux d'accroissement aussi élevé que 700 pour cent. Si la sécurité interne n'avait connu, pour sa part, qu'une fraction seulement de ce taux d'accroissement, il pourrait bien en résulter qu'aujourd'hui, au Canada, le nombre de personnes au service de la sécurité privée a pris une avance significative sur celui des policiers publics.

Alors que les personnes au service de la sécurité privée (définies au sens large pour inclure les forces de sécurité du gouvernement) surpassaient en nombre les effectifs de la police publique en 1971, les dépenses pour la sécurité privée furent au cours de cette même année, bien moindres que celles destinées à la police publique. Il en coata 646,9 millions \$ aux Canadiens en 1971 pour assurer les services de police publique. Pour la même période, Farnell et Shearing évaluent à 254,9 millions \$ le coût des salaires de la sécurité privée. Bien qu'ils signalent que cette évaluation sous-estime probablement les dépenses salariales et même si ces chiffres ne peuvent être comparés directement à ceux de la police publique (qui ne se limitent pas aux seules dépenses salariales), il demeure clair que le coût total, et le coût par homme, de la sécurité privée étaient considérablement moins élevés que ceux de la police publique.

\section{Diagramme $\mathrm{n}^{\circ} 2$}

40\% Division 10:

(Services communautaires, commerciaux et personnels)

29\% Division 11:

(Administration publique)

13\% Division 5:

(Manufacture)

6\% Division 7:

(Transport et Communications)

4\% Division 8:

(Commerce)

3\% Division 6:

(Construction)

2\% Division 9:

(Finance, Assurance, Immobilier) 


\section{CONCLUSION: LA RÉVOLUTION TRANQUILLE}

Il semble y avoir peu de doute sur le fait que la sécurité privée s'est installée à demeure et qu'elle constituera une pièce de plus en plus importante de l'appareil du contrôle social. Qu'est-ce à dire? Quel sera l'impact de la sécurité privée sur la qualité de la vie, dans la société canadienne? La réaction immédiate à ces interrogations pourrait bien être qu'elle semble avoir eu très peu d'effets. Comme nous l'avons mentionné au début, il y a peu de vrai à dire cela. La sécurité privée s'est développée tranquillement. Sa présence accrue a été à peine remarquée. Après un examen plus attentif, ces changements discrets s'avèrent toutefois porteurs d'implications révolutionnaires. Avec le développement de la sécurité privée, le foyer du contrôle social s'est déplacé du secteur public vers le secteur privé, des gouvernements vers les corporations et des groupes locaux vers les individus. Cette situation souleve des questions cruciales, que l'on commence à peine à distinguer. Un tel déplacement représentet-il, comme le suggèrent Freedman et Stenning (1977, p. 53) une forme de désenchantement avec la police publique? Signifie-t-il un accroissement de l'influence d'intérêts privés locaux aux dépens de "l'intérêt public» en ce qui a trait au contrôle social? Cela est-il une bonne ou une mauvaise chose? La "privatisation" de la police faitelle partie d'un mouvement général que l'on commence à percevoir dans d'autres domaines des services publics tels que l'éducation et les postes? Si tel est le cas, que signifie ce phénomène pour le rôle d'un gouvernement central? Est-ce qu'au Canada les pressions politiques relatives à la constitution et l'autonomie des provinces sont des phénomènes qui ont un rapport avec nos hypothèses sur l'érosion du rôle des gouvernements dans les processus de contrôle social? Quel effet le développement de la sécurité privée a-t-il eu sur le pouvoir des corporations, et plus spécialement des multinationales, vis-à-vis de l'individu et de l'État? A-t-il accentué leur stature et leur pouvoir, comme le suggère Friedenberg (1975)?

Telles sont les questions que soulèvent les faits concernant la sécurité privée. Le programme de recherche du Centre de criminologie de l'Université de Toronto ayant franchi l'étape initiale de la colligation des faits, c'est dorénavant vers la résolution de ces problèmes que s'oriente la recherche. 
TABLEAU I

Répartition du personnel de la sécurité privée en 1971, selon la Province et le type d'organisation

\begin{tabular}{|c|c|c|c|c|c|c|c|c|}
\hline \multirow[b]{2}{*}{ Province } & \multicolumn{2}{|c|}{ Sécurité à contrat } & \multicolumn{4}{|c|}{ Sécurité interne } & \multirow[b]{2}{*}{$\begin{array}{c}\text { Sécurité } \\
\text { privée } \\
\text { total }\end{array}$} & \multirow[b]{2}{*}{$\begin{array}{l}\% \text { du } \\
\text { total }\end{array}$} \\
\hline & total & $\begin{array}{l}\% \text { du } \\
\text { total }\end{array}$ & $\begin{array}{l}\text { Privé } \\
\text { total }\end{array}$ & $\begin{array}{l}\% \mathrm{du} \\
\text { total }\end{array}$ & $\begin{array}{l}\text { Gouver- } \\
\text { nement } \\
\text { total }\end{array}$ & $\begin{array}{l}\% \text { du } \\
\text { total }\end{array}$ & & \\
\hline Terre-Neuve & 100 & 9 & 760 & 3 & 200 & 1,4 & 1060 & 2,1 \\
\hline Île-du-Prince-Édouard & 60 & 0,6 & 45 & 0,2 & 40 & 0,2 & 145 & 0,2 \\
\hline Nouvelle-Écosse & 570 & 5 & 880 & 3,4 & 460 & 3,4 & 1910 & 4 \\
\hline Nouveau-Brunswick & 435 & 4 & 845 & 3,3 & 410 & 3 & 1690 & 3,3 \\
\hline Québec & 3295 & 28,5 & 9170 & 36,4 & 4250 & 31,5 & 16715 & 33,2 \\
\hline Ontario & 4780 & 41,4 & 8325 & 33 & 4785 & 35,4 & 17890 & 36 \\
\hline Manitoba & 510 & 4,4 & 750 & 3 & 520 & 4 & 1780 & 3,5 \\
\hline Saskatchewan & 255 & 2,2 & 600 & 2,3 & 400 & 3 & 1255 & 2,5 \\
\hline Alberta & 675 & 5,8 & 1285 & 5,1 & 865 & 6,4 & 2825 & 6 \\
\hline Colombie Britannique & 850 & 7,3 & 2475 & 10 & 1435 & 106 & 4760 & 9,4 \\
\hline \multicolumn{9}{|l|}{ Yukon, } \\
\hline Territoires du Nord-Ouest & 10 & 0,8 & 45 & 0,2 & 115 & 0,8 & 170 & 0,3 \\
\hline Total & 11540 & 100 & 25180 & 100 & 13480 & 100 & 50200 & 100 \\
\hline
\end{tabular}

Source: Groupe de diffusion des données, Statistique Canada.

* Puisque les donncés de Statistique Canada sont soumises à une procédure stochastique d'arrondissage des chiffres, les totaux ne correspondent pas nécessairement à la somme des chiffres arrondis des répartitions. 


\section{BIBLIOGRAPHIE}

BECKER, Theodore M. (1974), «The Place of Private Police in Society: An Area of Research for the Social Sciences", Social Problems, vol. 21, nº 3, January.

CHAMBLISS, W.J. et R.B. SEIDMAN (1970), Sociology of Law: A Research Bibliography, Betkeley, Calif., Glendessary Press.

CRITCHLEY, T.A. (1967), A History of Police in England and Wales, 1900-1966, London, Constable.

DRAPER, Hilary (1978), Private Police, Harmondsworth, England, Penguin Books.

FARNELL, Margaret and C.D. SHEARING (1977), Private Security: An Examination of Canadian Statistics, 1961-1971, Toronto, Centre of Criminology, University of Toronto.

FREEDMAN, David J. and Philip C. STENNING (1977), Private Security, Police and the Law in Canada, Toronto, Centre of Criminology, University of Toronto.

FRIEDENBERG, E.Z. (1975), The Disposal of Liberty and Other Industrial Wast, New York, Doubleday.

JEFFRIES, Fern (1977), Private Policing: An Examination of In-House Security Operations, 1977. Toronto, Centre of Criminology, University of Toronto.

JEFFRIES, Fern (ed.) (1974), Private Policing and Security in Canada: $A$ Workshop, 1973. Report of the Proceedings, Toronto, Centre of Criminology, University of Toronto.

KAKALIK, J.S. and S. WILDHORN (1972), Private Police in the United States, vol. IV, The Law and Private Police, Santa Monica, Calif., Rand Corporation.

KAKALIK, J.S. and S. WILDHORN (1971), The Private Police: Security and Danger, New York, Crone Russak.

KEETON, G.W. (1975), Keeping the Peace, Chichester, Barry Rose.

LEWIS, Roy (1976), A Force for the Future: The Role of the Police in the Next Ten Years, London, Temple Smith.

MAITLAND, F. (1913), The Constitutional History of England, Cambridge, University Press, 1913.

MORAND, D.R. (1976), The Royal Commission into Metropolitain Toronto Police Practices, Toronto, The Commission.

PLEECE, Sydney (1972), "The Nature and Potential of the Security Industry», Police Journal, vol. XLIV n ${ }^{\circ}$ 1, January-March, p. 41.

POST, R.S. (1971), «Relations with Private Police Services», The Police Chief, March, p. 54-56.

REISS, Albert J. Jr. (1971), The Police and the Public, New Haven, Conn., and London, University Press.

SHEARING, Clifford D. (1974), «Dial-A-Cop: A Study of Police Mobilization», Crime Prevention and Social Defense, ed. R.L. Akers et E. Sagari, New York, Praeger.

STENNING, Philip C. and M.F. CORNISH (1975), The Legal Regulation and Control of Private Policing and Security in Canada: A Working Paper, Toronto, Centre of Criminology, University of Toronto. 
STENNING, Philip C. and Clifford D. SHEARING (1979), Search and Seizure: Power of Private Security Personnel (A Study Paper prepared fot the Law Reform Commission of Canada), Ottawa, Ministry of Supply and Services.

STENNING, P.C. and C.D. SHEARING (à paraitre), «Private Security and Private Justice: Doing Justice to Justice», British Journal of Law and Society.

STINCHCOMBE, A.L. (1963), «Institutions of Privacy in the Determination of Police Administrative Practice", American Journal of Sociology, vol. 69, September, p. 150.

WILES, Paul and F.H. McCLINTOCK (eds.) (1972), «The Security Industry in the United Kingdom; Papers Presented to the Cropwood Round-Table Conference, July $1971 n$, Cambridge, Institue of Criminology. 